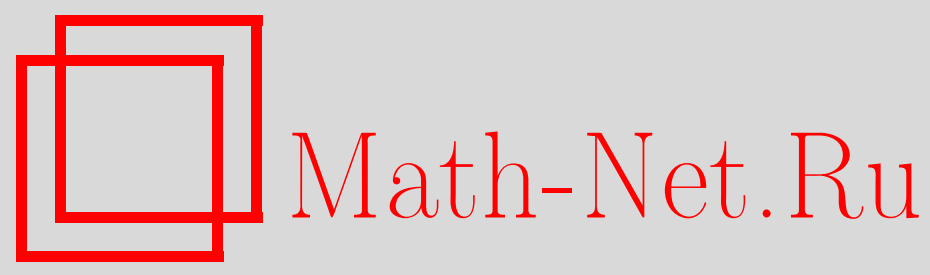

М. Ф. Сухинин, K вопросу о беллмановском подходе в теории оптимального управления, Матем. заметки, 1999, том 66, выпуск 5, 770-776

DOI: https://doi.org/10.4213/mzm1220

Использование Общероссийского математического портала Math-Net.Ru подразумевает, что вы прочитали и согласны с пользовательским соглашением http://www.mathnet.ru/rus/agreement

Параметры загрузки:

IP : 18.234 .197 .8

26 апреля 2023 г., 13:23:04

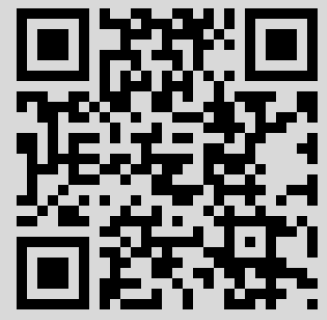




\title{
К ВОПРОСУ О БЕЛЛМАНОВСКОМ ПОДХОДЕ В ТЕОРИИ ОПТИМАЛЬНОГО УПРАВЛЕНИЯ
}

\author{
М. Ф. Сухинин
}

Усилена ранее опубликованная теорема автора, что позволило получить более информативное необходимое условие экстремума в задачах оптимального управления. Результат проиллюстрирован на простейшей задаче оптимального управления.

Библиография: 8 названий.

1. Известно, что уравнение Беллмана в теории оптимальных процессов требует для своего вывода наличия весьма ограничительных предположений, не выполняющихся во многих простых задачах (см., например, $[1$, с. 85]), хотя основная идея Беллмана не связана с этими предположениями. Здесь излагается другая форма реализации этой идеи, свободная от этого недостатка. Дело в том, что для реализации идеи Беллмана достаточно наличия свойства, близкого к существованию производной по определенному направлению у соответствующей функции векторного аргумента (именно эта идея здесь и развивается), тогда как для записи уравнения Беллмана требуется существование градиента этой функции, что является более ограничительным требованием и тем самым сужает класс задач, в действительности охватьваемых беллмановским подходом. Приведенньй здесь результат усиливает ранее опубликованньй результат автора в этом направлении ([2], [3, с. 270]). Иной взгляд на уравнение Беллмана изложен в [4]-[6].

2. Пусть $X$ - банахово пространство, $B=\{x \in X \mid\|x\| \leqslant 1\}, W$ - непустое множество в $X$. Для $l \in X, x \in W$ и $\varphi: W \longrightarrow \mathbb{R}$ положим

$$
D S_{l} \varphi(x)=\bigcap_{\varepsilon>0} \overline{\bigcup_{0<\delta<\varepsilon} \delta^{-1}(\varphi\{[x+\delta(l+\varepsilon B)] \cap W\}-\varphi(x))},
$$

где черта означает замыкание в $X$, и назовем $D S_{l} \varphi(x)$ дифференииальны.м множеством функции $\varphi$ в точке $x$ по элементу $l$. Если $D S_{l} \varphi(x)$ состоит из одного элемента, то $\varphi$ назьвается равномерно дифферениируемым в точке $x$ по направлению $l$ (при $l \neq 0$ ) (определение равномерной дифференцируемости по направлению для отображений топологических векторных пространств дано в $[7$, c. 220] для того случая, когда $x \in \operatorname{int} W)$. При этом единственный элемент множества $D S_{l} \varphi(x)$ обозначается через $\varphi^{\prime}(x, l)$.

3. Пусть теперь задано отображение $P: W \longrightarrow 2^{X} \backslash\{\varnothing\}$, т.е. многозначное отображение $W$ в $X$ с непустым образом $P(x)(\forall x \in W)$, а поведение некоторой системы описывается функцией $x:[0, T[X(0<T \leqslant \infty)$, удовлетворяющей дифференциальному включению

$$
\dot{x}(t) \in P(x(t))
$$


и начальному условию

$$
x(0)=x_{0} \in W
$$

(как обычно, считается, что функция $x(\cdot)$ локально абсолютно непрерывна и существует $\dot{x}(t)$ почти для каждого $t$, причем $x(t)=x_{0}+\int_{0}^{t} \dot{x}(s) d s \in W$, где интеграл понимается в смысле Бохнера). Далее, пусть $x_{1}$ - фиксированный элемент из $W$. Обозначим через $D(\omega)$ множество тех $x_{0} \in W$, для которых существует траектория, удовлетворяющая (3.1) и (3.2), двигаясь вдоль которой, наша система перейдет из точки $x_{0}$ в точку $x_{1}$ за время $t\left(x_{0}, x(\cdot)\right)<T$, и положим $\omega\left(x_{0}\right)=\sup \left(-t\left(x_{0}, x(\cdot)\right)\right)$ по всем таким траекториям. При этом $D(\omega)$ - область определения функции $\omega$.

4. Пусть $x \in D(\omega)$. Элемент $l \in P(x)$ назовем допустимым для $x$, если

1) $l \neq 0$;

2) $\forall \varepsilon>0 \exists \delta \in] 0, \varepsilon\left[\forall x^{\prime} \in(x+] 0, \delta[\cdot(l+\delta B)) \cap D(\omega) \exists t^{\prime} \in\right] 0, \varepsilon\left[\exists x(\cdot) \in W_{1}^{1}\left(\left[0, t^{\prime}\right], X\right)\right.$ :

a) $\dot{x}(t) \in P(x(t))$ почти для каждого $t \in\left[0, t^{\prime}\right]$;

б) $x(0)=x$;

в) $x\left(t^{\prime}\right) \in D(\omega)$;

г) $\omega\left(x^{\prime}\right)-\omega\left(x\left(t^{\prime}\right)\right) \leqslant t^{\prime} \varepsilon$

д) $\left\|x^{\prime}-x-l t^{\prime}\right\| \leqslant t^{\prime} \varepsilon$.

Здесь $W_{1}^{1}\left(\left[0, t^{\prime}\right], X\right)$ - множество функций, представимых в виде $\Phi(t)=a+\int_{0}^{t} \varphi(s) d s$, где $a \in X, \mathrm{a} \varphi:\left[0, t^{\prime}\right] \longrightarrow X-$ интегрируемая по Бохнеру функция. Множество допустимых для $x \in D(\omega)$ элементов обозначим через $U(x)$.

5. ЗАмЕЧАНИЕ. В [2], [3, с. 270] вместо условий в) и г) стоит условие $x\left(t^{\prime}\right)=x^{\prime}$. Замена этого условия на условия в) и г) расширяет множество $U(x)$ и позволяет усилить упомянутые результаты.

6. Теорема. Для каждого $x \in D(\omega)$ такого, ито $U(x) \neq \varnothing$, имеет место неравенство

$$
\sup _{l \in U(x)} \sup D S_{l} \omega(x) \leqslant 1
$$

(при условии, что $\sup \varnothing=-\infty)$.

ДокАЗАТЕЛЬСтво. Допустим, что

$$
\sup _{l \in U(x)} \sup D S_{l} \omega(x)>1
$$

Тогда

$$
\begin{gathered}
\exists \sigma>0 \exists l \in U(x) \forall \xi>0 \exists \eta \in] 0, \xi[\exists h \in B: \\
{[x+\eta(l+\xi h) \in D(\omega)] \wedge[\omega(x+\eta(l+\xi h))-\omega(x) \geqslant(1+2 \sigma) \eta] .}
\end{gathered}
$$

Придадим $\xi$ последовательность значений $\xi_{n}=n^{-1}, n \in \mathbb{N}$. Для каждого $n$ найдем $\left.\eta_{n} \in\right] 0, \xi_{n}\left[\right.$ и $h_{n} \in B$ так, чтобы $x+\eta_{n}\left(l+\xi_{n} h_{n}\right) \in D(\omega)$ и

$$
\omega\left(x+\eta_{n}\left(l+\xi_{n} h_{n}\right)\right)-\omega(x) \geqslant(1+2 \sigma) \eta_{n} .
$$


Положим $\varepsilon_{k}=k^{-1}, k \in \mathbb{N}$. Для $\varepsilon=\varepsilon_{k}$ найдем $\left.\delta=\delta_{k} \in\right] 0, \varepsilon_{k}$ [ так, чтобы выполнялось 2) в п. 4 , начиная с $\forall x^{\prime}$. По каждому $k$ найдем $n_{k} \geqslant k$ так, чтобы $\xi_{n_{k}}<\delta_{k}$ и $\eta_{n_{k}}<$ $\delta_{k}$. Для $x_{k}^{\prime}=x+\eta_{n_{k}}\left(l+\xi_{n_{k}} h_{n_{k}}\right)$ найдем $\left.t_{k} \in\right] 0, \varepsilon_{k}\left[\right.$ и $x_{k}(\cdot) \in W_{1}^{1}\left(\left[0, t_{k}\right], X\right)$ так, чтобы $\dot{x}_{k}(t) \in P\left(x_{k}(t)\right)$ почти для каждого $t \in\left[0, t_{k}\right], x_{k}(0)=x, \omega\left(x_{k}^{\prime}\right)-\omega\left(x_{k}\left(t_{k}\right)\right) \leqslant t_{k} \varepsilon_{k}$ и $\left\|x_{k}^{\prime}-x-l t_{k}\right\| \leqslant t_{k} \varepsilon_{k}$ (это можно сделать согласно 2)). Заметим, что $x_{k}^{\prime}-x-l t_{k}=t_{k} \varepsilon_{k} h_{k}^{\prime}$ для некоторого $h_{k}^{\prime} \in B$. Докажем, что $\eta_{n_{k}}^{-1} t_{k} \rightarrow 1$ при $k \rightarrow \infty$. Сначала установим ограниченность последовательности $\eta_{n_{k}}^{-1} t_{k}$. Допустим, что она неограничена. Тогда, переходя, если нужно, к подпоследовательности и переобозначив ее, можно считать, что $\eta_{n_{k}}^{-1} t_{k} \rightarrow \infty$. В этом случае

$$
\begin{aligned}
t_{k}^{-1} l\left(\eta_{n_{k}}-t_{k}\right) & =t_{k}^{-1}\left[\left(x_{k}^{\prime}-x-\eta_{n_{k}} \xi_{n_{k}} h_{n_{k}}\right)-\left(x_{k}^{\prime}-x-t_{k} \varepsilon_{k} h_{k}^{\prime}\right)\right] \\
& =t_{k}^{-1}\left(t_{k} \varepsilon_{k} h_{k}^{\prime}-\eta_{n_{k}} \xi_{n_{k}} h_{n_{k}}\right)=\varepsilon_{k} h_{k}^{\prime}-\left(t_{k}^{-1} \eta_{n_{k}}\right) \xi_{n_{k}} h_{n_{k}} \rightarrow 0
\end{aligned}
$$

при $k \rightarrow \infty$. С другой стороны, $t_{k}^{-1} l\left(\eta_{n_{k}}-t_{k}\right)=l\left(\eta_{n_{k}} t_{k}^{-1}-1\right) \rightarrow-l \neq 0$. Полученное противоречие показывает, что последовательность $\eta_{n_{k}}^{-1} t_{k}$ ограничена. Аналогично, предположив, что $t_{k}^{-1} \eta_{n_{k}} \rightarrow \infty$, получим, что

$$
\eta_{n_{k}}^{-1} l\left(\eta_{n_{k}}-t_{k}\right)=\eta_{n_{k}}^{-1}\left(t_{k} \varepsilon_{k} h_{k}^{\prime}-\eta_{n_{k}} \xi_{n_{k}} h_{n_{k}}\right)=\left(\eta_{n_{k}}^{-1} t_{k}\right) \varepsilon_{k} h_{k}^{\prime}-\xi_{n_{k}} h_{n_{k}} \rightarrow 0
$$

тогда как $\eta_{n_{k}}^{-1} l\left(\eta_{n_{k}}-t_{k}\right)=l\left(1-\eta_{n_{k}}^{-1} t_{k}\right) \rightarrow l \neq 0$. Это означает, что последовательность $t_{k}^{-1} \eta_{n_{k}}$ также ограничена. Но тогда

$$
l\left(\eta_{n_{k}} t_{k}^{-1}-1\right)=t_{k}^{-1} l\left(\eta_{n_{k}}-t_{k}\right)=\varepsilon_{k} h_{k}^{\prime}-\left(t_{k}^{-1} \eta_{n_{k}}\right) \xi_{n_{k}} h_{n_{k}} \rightarrow 0
$$

а отсюда следует, что $\eta_{n_{k}} t_{k}^{-1} \rightarrow 1$. Поэтому для достаточно больших $k$ будет вьполняться неравенство $(1+2 \sigma) \eta_{n_{k}}>\left(1+\sigma+\varepsilon_{k}\right) t_{k}$. Заметим, что $t_{k}-$ время движения по траектории $x_{k}(\cdot)$ из точки $x$ в точку $x_{k}\left(t_{k}\right)$. Поэтому если система будет двигаться из точки $x$ в точку $x_{k}\left(t_{k}\right)$ вдоль траектории $x_{k}(\cdot)$, а из точки $x_{k}\left(t_{k}\right)$ в точку $x_{1}$ по траектории, время движения по которой $\tilde{t}_{k}$ меньше, чем $-\omega\left(x_{k}\left(t_{k}\right)\right)+\sigma t_{k}$ (такая траектория существует по определению функции $\omega$ ), то общее время движения из точки $x$ в точку $x_{1}$ по такой составной траектории будет равно

$$
\begin{aligned}
t_{k}+\tilde{t}_{k} & <t_{k}-\omega\left(x_{k}\left(t_{k}\right)\right)+\sigma t_{k} \leqslant-\omega\left(x_{k}^{\prime}\right)+t_{k} \varepsilon_{k}+(1+\sigma) t_{k} \\
& <-\omega\left(x_{k}^{\prime}\right)+(1+2 \sigma) \eta_{n_{k}} \leqslant-\omega(x)
\end{aligned}
$$

согласно (6.1), а это противоречит определению функции $\omega$. Теорема доказана.

7. Пусть теперь $x_{0} \in D(\omega), x_{0} \neq x_{1}, \bar{x}(\cdot)$ - оптимальная по быстродействию траектория, являющаяся решением задачи $(3.1),(3.2)$ и соединяющая точку $x_{0}$ с точкой $x_{1}$. В этом случае $-\omega\left(x_{0}\right)$ - время движения по траектории $\bar{x}(\cdot)$. Заметим, что при $t \neq t^{\prime}$ $\left(t\right.$ и $\left.t^{\prime} \leqslant-\omega\left(x_{0}\right)\right)$ будет $\bar{x}(t) \neq \bar{x}\left(t^{\prime}\right)$, т.е. время $t$ (при $\left.t \leqslant-\omega\left(x_{0}\right)\right)$ движения системы из точки $x_{0}=\bar{x}(0)$ в точку $\bar{x}(t)$ по траектории $\bar{x}(\cdot)$ является однозначной функцией $t(x)$ точки $x$ на этой траектории (в силу оптимальности). Пусть

$$
M(\bar{x}(\cdot))=\left\{x \in \bigcup_{0 \leqslant t<-\omega\left(x_{0}\right)}\{\bar{x}(t)\}\left|\dot{x}_{r}(t)\right|_{t=t(x)} \in P(x)\right\}
$$

(здесь $\dot{\bar{x}}_{r}(t)$ - правая производная). Для $x \in M(\bar{x}(\cdot))$ положим $\bar{l}(x)=\left.\dot{\bar{x}}_{r}(t)\right|_{t=t(x)}$. 
8. Teоpema. Пyсmb $x \in M(\bar{x}(\cdot))$. Torda $1 \in D S_{\bar{l}(x)} \omega(x)$.

ДокАЗАТЕЛЬСтво. Пусть $0<t_{n}<-\omega(x)$ и $t_{n} \rightarrow 0$. Тогда $\bar{x}\left(t(x)+t_{n}\right)=x+$ $\bar{l}(x) t_{n}+t_{n} \varepsilon_{n} h_{n} \in D(\omega)$, где $\varepsilon_{n}>0, \varepsilon_{n} \rightarrow 0$ и $h_{n} \in B$, поскольку $\left.\dot{\bar{x}}_{r}(t)\right|_{t=t(x)}=\bar{l}(x)$. Далее, $-\omega\left(\bar{x}\left(t(x)+t_{n}\right)\right)=-\omega(x)-t_{n}$, в силу того что кусок оптимальной траектории является оптимальной траекторией для конщов этого куска. Поэтому

$\forall \varepsilon>0 \exists N \in \mathbb{N} \forall n>N$ :

$$
\begin{aligned}
& \bigcup_{0<\delta<\varepsilon} \delta^{-1}[\omega(\{x+\delta[\bar{l}(x)+\varepsilon B]\} \cap D(\omega))-\omega(x)] \\
& \quad \ni t_{n}^{-1}\left(\omega\left\{x+t_{n}\left[\bar{l}(x)+\varepsilon_{n} h_{n}\right]\right\}-\omega(x)\right)=t_{n}^{-1}\left(\omega\left\{\bar{x}\left(t(x)+t_{n}\right)\right\}-\omega(x)\right) \\
& \quad=t_{n}^{-1}\left(\omega(x)+t_{n}-\omega(x)\right)=1,
\end{aligned}
$$

откуда следует, что $1 \in D S_{\bar{l}(x)} \omega(x)$. Теорема доказана.

9. СлеДСтвиЕ. Пусть $x \in M(\bar{x}(\cdot)) u \bar{l}(x) \in U(x)$. Тогда

$$
\sup _{l \in U(x)} \sup D S_{l} \omega(x)=\sup D S_{\bar{l}(x)} \omega(x)=1 .
$$

Это вытекает из пп. 6 и 8 , поскольку

$$
1 \leqslant \sup D S_{\bar{l}(x)} \omega(x) \leqslant \sup _{l \in U(x)} \sup D S_{l} \omega(x) \leqslant 1 .
$$

10. СлЕДСтвИЕ. Пусть $x \in M(\bar{x}(\cdot))$, функиия $\omega$ равномерно дифференцируема в точке $x$ по каждому направлению из некоторого множества $\widetilde{U}(x) \subset U(x)(c \mathcal{M}$. n. 2) $u \bar{l}(x) \in \widetilde{U}(x)$. Тогда

$$
\sup _{l \in \widetilde{U}(x)} \omega^{\prime}(x, l)=\omega^{\prime}(x, \bar{l}(x))=1
$$

Это вытекает из п. 9 и определения равномерной дифференцируемости по направлению (см. п. 2).

11. Рассмотрим простейшую задачу оптимального управления, т.е. задачу приведения системы

$$
\dot{x}^{1}=x^{2}, \quad \dot{x}^{2}=u(t)
$$

из начального состояния $\left(x_{0}^{1}, x_{0}^{2}\right)$ в начало координат за минимальное время за счет выбора измеримой функции $u(\cdot)$, удовлетворяющей условию

$$
|u(t)| \leqslant 1 .
$$

Известно [1, с. 31], что оптимальньми траекториями задачи (11.1), (11.2) являются куски парабол $\mathcal{L}_{ \pm}: x^{1}= \pm 2\left(x^{2}\right)^{2}+C$, получаемых при интегрировании системы (11.1) при $u(t)= \pm 1$, а линией переключения является $\Gamma=\Gamma_{+} \cup \Gamma_{-}$, где

$$
\begin{aligned}
& \Gamma_{+}=\left\{\left(x^{1}, x^{2}\right) \mid x^{1}=2^{-1}\left(x^{2}\right)^{2}, x^{2} \leqslant 0\right\}, \\
& \Gamma_{-}=\left\{\left(x^{1}, x^{2}\right) \mid x^{1}=-2^{-1}\left(x^{2}\right)^{2}, x^{2} \geqslant 0\right\} .
\end{aligned}
$$




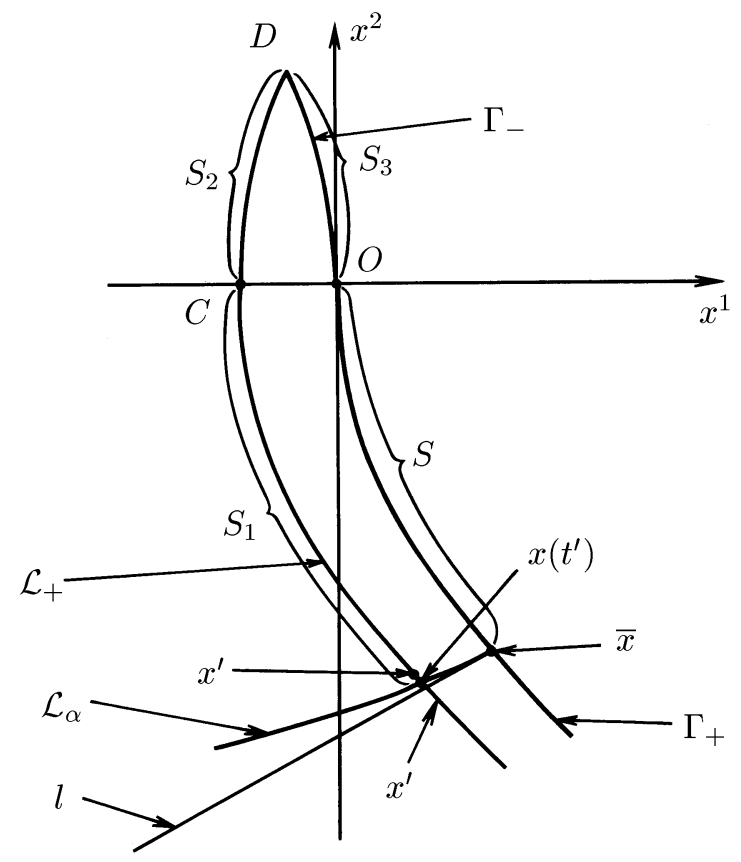

Рис. 1

Перепишем задачу $(11.1),(11.2)$ в терминах пп. 3,4 . Здесь $X=W=\mathbb{R}^{2}, P(x)=$ $\left(x^{2},[-1,1]\right), D(\omega)=\mathbb{R}^{2}, x_{1}=0$. Покажем, что $U(x)=\left(x^{2},[-1,1]\right) \backslash\{0\}$ при $x \in D(\omega) \backslash$ $\{0\}$. Пусть сначала точка $\bar{x}=\left(\bar{x}^{1}, \bar{x}^{2}\right) \neq 0$ лежит, например, на $\Gamma_{+}, l=\left(\bar{x}^{2}, \alpha\right)$, где $\alpha \in[-1,1]$, и $l \neq 0$. Фиксируем $\varepsilon>0$. Тогда задача

$$
\dot{x}^{1}=x^{2}, \quad \dot{x}^{2}=\alpha, \quad x^{1}(0)=\bar{x}^{1}, \quad x^{2}(0)=\bar{x}^{2}
$$

имеет единственное решение $\left(x^{1}(t), x^{2}(t)\right)$ на $\left[0,+\infty\left[\right.\right.$, где $x^{1}(t)=\alpha t^{2} / 2+\bar{x}^{2} t+\bar{x}^{1}$ и $x^{2}=\alpha t+\bar{x}^{2}$. Обозначим ее траекторию через $\mathcal{L}_{\alpha}$. Возможны два случая: 1$) \alpha \in[-1,1[$, 2) $\alpha=1$. Рассмотрим каждый из них.

1) Пусть $\alpha \in\left[-1,1\left[\right.\right.$. В этом случае если $\left.x^{\prime} \in \bar{x}+\right] 0, \delta[\cdot(l+\delta B)$ при достаточно малом $\delta \in] 0, \varepsilon\left[\right.$, то в качестве $x\left(t^{\prime}\right)$ можно взять точку пересечения линии из $\mathcal{L}_{+}$, проходящей через точку $x^{\prime}$, с линией $\mathcal{L}_{\alpha}$. Ввиду того что кусок линии из $\mathcal{L}_{+}$, соединяющей точки $x^{\prime}$ и $x\left(t^{\prime}\right)$, имеет длину порядка $O\left(\delta t^{\prime}\right)$ (в силу того что $\alpha<1$ и, следовательно, линии из $\mathcal{L}_{+}$ пересекаются с $\mathcal{L}_{\alpha}$ вблизи точки $\bar{x}$ под отделенным от нуля углом), а модули скоростей движения по оптимальным траекториям вблизи точки $\bar{x}$ имеют порядок $v(\bar{x})\left(1+O\left(\delta t^{\prime}\right)\right)$, где $v(\bar{x})$ - модуль скорости движения по $\Gamma_{+}$в точке $\bar{x}$, время движения из точки $x\left(t^{\prime}\right)$ в точку $x^{\prime}$, если $x^{\prime}$ лежит над $\mathcal{L}_{\alpha}$ (см. рис. 1), имеет порядок $O\left(\delta t^{\prime}\right) /\left(v(\bar{x})\left(1+O\left(\delta t^{\prime}\right)\right)\right)=$ $O\left(\delta t^{\prime}\right)$. Поэтому при достаточно малом $\left.\delta \in\right] 0, \varepsilon[$ вьполняется условие г) из п. 4 . Если же $x^{\prime}$ лежит под $\mathcal{L}_{\alpha}$ или на $\mathcal{L}_{\alpha}$, то $\omega\left(x^{\prime}\right) \leqslant \omega\left(x\left(t^{\prime}\right)\right)$ и г) тем более выполняется. Поэтому $l \in U(\bar{x})$. Далее, время движения по траекториям $C D$ и $D 0$ (см. рис. 1 ) равно

$$
\frac{S_{2}}{v(0)\left(1+O\left(\delta t^{\prime}\right)\right)}+\frac{S_{3}}{v(0)\left(1+O\left(\delta t^{\prime}\right)\right)} \geqslant \frac{2 \beta\left(t^{\prime}\right)^{1 / 2}}{1+O\left(\delta t^{\prime}\right)}>\beta\left(t^{\prime}\right)^{1 / 2}
$$


при некотором $\beta>0$. Например, для $\Gamma_{-}$в окрестности нуля $x^{2}=\left(-2 x^{1}\right)^{1 / 2} \geqslant \eta\left(\delta t^{\prime}\right)^{1 / 2}$ при некотором $\eta>0$, где $\left(x^{1}, x^{2}\right)=D$ - точка пересечения линии из $\mathcal{L}_{+}$, проходящей через $x\left(t^{\prime}\right)$, с $\Gamma_{-}$. Наконец, время движения по линии из $\mathcal{L}_{+}$от $x\left(t^{\prime}\right)$ до $C$ отличается от времени движения по $\Gamma_{+}$от $\bar{x}$ до 0 на величину порядка $O\left(\delta t^{\prime}\right)$ по теореме о непрерьвной дифференцируемости и, следовательно, липшицевости решения задачи Коши по начальньм данным и параметру, поскольку

$$
\frac{S}{\bar{v}}-\frac{S\left(1+O\left(\delta t^{\prime}\right)\right)}{\bar{v}\left(1+O\left(\delta t^{\prime}\right)\right)}=O\left(\delta t^{\prime}\right),
$$

где $\bar{v}$ - средняя линейная скорость движения от $\bar{x}$ до 0 по $\Gamma_{+}$, а $S$ - длина дуги $\bar{x} 0$ на $\Gamma_{+}$. Поэтому

$$
\begin{aligned}
\frac{\left(\omega\left(x^{\prime}\right)-\omega(\bar{x})\right)}{t^{\prime}} & \leqslant \frac{\omega\left(x\left(t^{\prime}\right)\right)+t^{\prime} \varepsilon-\omega(\bar{x})}{t^{\prime}} \\
& \leqslant \frac{\omega(\bar{x})-\beta\left(t^{\prime}\right)^{1 / 2}\left(1+O\left(\delta t^{\prime}\right)\right)+O\left(\delta t^{\prime}\right)+t^{\prime} \varepsilon-\omega(\bar{x})}{t^{\prime}} \\
& \leqslant-\beta\left(t^{\prime}\right)^{-1 / 2}\left(1+O\left(\delta t^{\prime}\right)\right)+\varepsilon+O(1) \rightarrow-\infty
\end{aligned}
$$

при $t^{\prime} \rightarrow+0$. Отсюда следует, что $D S_{l} \omega(\bar{x})=\varnothing$.

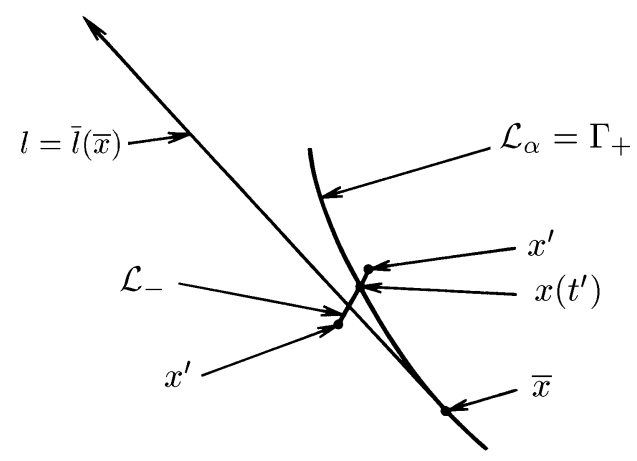

Рис. 2

2) Пусть $\alpha=1$. В этом случае если $\left.x^{\prime} \in \bar{x}+\right] 0, \delta[\cdot(l+\delta B)$ при достаточно малом $\delta \in] 0, \varepsilon\left[\right.$, то в качестве $x\left(t^{\prime}\right)$ можно взять точку пересечения линии из $\mathcal{L}_{-}$, проходящей через $x^{\prime}$, с $\mathcal{L}_{\alpha}=\Gamma_{+}$(см. рис. 2). Если $x^{\prime}$ лежит над $\mathcal{L}_{\alpha}$ или на $\mathcal{L}_{\alpha}$, то $\omega\left(x^{\prime}\right) \leqslant \omega\left(x\left(t^{\prime}\right)\right)$, если же $x^{\prime}$ лежит под $\mathcal{L}_{\alpha}$, то, как и в случае 1$)$, можно показать, что $\omega\left(x^{\prime}\right)<\omega\left(x\left(t^{\prime}\right)\right)-$ $\beta\left(\delta t^{\prime}\right)^{1 / 2}<\omega\left(x\left(t^{\prime}\right)\right)$ при достаточно малом $\left.\delta \in\right] 0, \varepsilon[$ и некотором $\beta>0$, ввиду того что линии из $\mathcal{L}_{-}$пересекаются с $\Gamma_{+}$вблизи точки $\bar{x}$ под отделенным от нуля углом. Следовательно, условие г) из п. 4 вьполнено при любом расположении $x^{\prime}$ относительно $\mathcal{L}_{\alpha}$. Поэтому $l \in U(\bar{x})$. Очевидно, что здесь $l=\bar{l}(\bar{x})$ (см. п. 7). Рассуждая так же, как и в случае 1$)$, получим, что $\left.\left.D S_{l} \omega(\bar{x})=\right]-\infty, 1\right]$, поскольку, очевидно, $D S_{l} \omega(\bar{x})$ связно и в силу п. $9 \sup D S_{\bar{l}(\bar{x})} \omega(\bar{x})=1$.

Заметим, что область определения классического решения уравнения Беллмана

$$
\sup _{|u| \leqslant 1}\left(\frac{\partial \omega(x)}{\partial x^{1}} x^{2}+\frac{\partial \omega(x)}{\partial x^{2}} u\right)=1
$$


не содержит $\Gamma$, поскольку $\nabla \omega(x)$ не существует.

Аналогично доказывается, что $U(x)=\left(x^{2},[-1,1]\right) \backslash\{0\}$ и $\bar{l}(x) \in U(x)$ вне линии переключения (если $\bar{x}$ лежит на оси $0 x^{1}$, то в качестве $x\left(t^{\prime}\right)$ можно взять точку пересечения перпендикуляра к $l$, проходящего через точку $x^{\prime}$, с линией $\left.\mathcal{L}_{\alpha}\right)$.

Похожая картина наблюдается в задаче управления гармоническим осциллятором [8, c. 18] $\dot{x}^{1}=x^{2}, \dot{x}^{2}=-x^{1}+u(t),|u(t)| \leqslant 1$, и в задаче управления под действием отталкивающей силы [8, с. 55] $\dot{x}^{1}=x^{2}, \dot{x}^{2}=k^{2} x^{1}-2 b x^{2}+u(t),|u(t)| \leqslant 1$, где $k^{2}>0$ и $b$ - константы. В том и другом случаях область определения классического решения уравнения Беллмана не содержит линии переключения ввиду несуществования $\nabla \omega(x)$, тогда как $\bar{l}(x) \in U(x)$ на линии переключения (за исключением особых точек этой линии) и, следовательно, необходимое условие оптимальности из п. 9 содержательно.

\section{СПИСОК ЦИТИРОВАННОЙ ЛИТЕРАТУРЫ}

[1] Понтрягин Л. С., Болтянский В. Г., Гамкрелидзе Р. В., Мищенко Е. Ф. Математическая теория оптимальных процессов. М.: Наука, 1976.

[2] Сухинин М. Ф. Об одном аналоге уравнения Беллмана // Матем. заметки. 1985. Т. 38. № 2. C. $267-271$.

[3] Сухинин М.Ф. Избранные главы нелинейного анализа. М.: Изд-во Российского университета дружбы народов, 1992.

[4] Болтянский В. Г. Достаточные условия оптимальности и обоснование метода динамического программирования // Изв. АН СССР. Сер. матем. 1964. Т. 28. №3. С. 481-514.

[5] Crandall M. G., Lions P.-L. Viscosity solutions of Hamilton-Jacobi equations // Trans. Amer. Math. Soc. 1983. V. 277. P. 1-42.

[6] Субботин А.И. Минимаксные неравенства и уравнения Гамильтона-Якоби. М.: Наука, 1991.

[7] Иоффе А. Д., Тихомиров В. М. Теория экстремальных задач. М.: Наука, 1974.

[8] Ли Э. В., Маркус Л. Основы теории оптимального управления. М.: Наука, 1972.

Российский университет дружбы народов, г. Москва

Поступило 30.07.1997

Исправленный вариант 24.05.1999 